Jiayu He

$\mathrm{Ye} \mathrm{Li}$

Yanqing Jiang

Yueming Li

$\mathrm{Li} A n$

http://dx.doi.org/10.21278/brod69210

\title{
PROPELLER FAULT DIAGNOSIS BASED ON A RANK PARTICLE FILTER FOR AUTONOMOUS UNDERWATER VEHICLES
}

UDC 629.5.035:629.58

Original scientific paper

\begin{abstract}
Summary
Rank particle filtering was applied to fault diagnosis technology. Control force and yaw moment losses that occurred in the corresponding degrees of freedom were estimated, and the trends of change were calculated by the multi-step-ahead prediction process in the rank particle filter. Based on the estimated results in the normal state, the situations involving failure were detected. To achieve this target, a mathematical model of the normal and faulty motion states of an underwater vehicle was first developed. Subsequently, the rank sample method combined with the particle filter was used, to obtain the importance probability density function of the autonomous underwater vehicle status. The rank particle filter obtained above realized the real-time state estimation and trend prediction of the motion state. A modified Bayesian algorithm was used to process the estimated control force and yaw moment losses in a given length of time. Based on the calculation results in the normal situations, a back propagation neural network was trained to obtain the diagnostic values. The condition of the propellers was determined based on the diagnostic values. Fault diagnosis simulation experiments were carried out using both the data obtained from the semi-physical simulation system with a hypothetical propeller failure, and the real sea trail data to verify the performance of the proposed algorithm. The results showed that the rank particle filter method could be applied to the propeller fault diagnosis of autonomous underwater vehicles, and solved the problem that arises when a single degree of freedom of the loss is utilized.
\end{abstract}

Key words: $\quad$ Autonomous Underwater Vehicle; Rank Particle Filter; Fault diagnosis; Propeller

\section{Introduction}

Deprived of a tether that would connect them to ships, the working state of autonomous underwater vehicles (AUVs) is difficult to observe in the underwater environment; therefore, AUVs must possess enough autonomy to accomplish missions [1]. For such vehicles to be capable of accomplishing missions, many advanced control systems are being widely applied to the guidance and control of these unmanned vehicles [2][3]. Without proper steering, a ship 
may lose its control under harsh conditions [4]. The underwater environment is even more complicated and variable, and frequently has unpredictable effects on AUVs. Such effects include minor and severe effects, where a minor effect may lead to the failure of a task, and a severe effect may lead to the damage, and even the loss of the AUV. For example, the loss accidents of KAIKO and Nereus [5][6] was regrettable, which brought significant losses to the research development of the two study teams. As described above, improvements in the AUV's operating ability increased the complexity of the AUV system [7][8], and the AUV's safety and security have received increasing attention. Therefore, the fault diagnosis of propulsion, sensors, and other equipment subsystems of the AUVs is required [9].

According to different classification criteria, fault types can be divided into different types and regions. As the manipulating operation of an AUV depends on its propellers, a fault in a propeller may lead to a failure in the underwater task, or even the eventual loss of the vehicle. For example, an AUV controlled by double stern propellers may encounter a situation where the propeller can output the desired thrust at low speed, but cannot output the desired thrust that exceeds a certain value. This failure affects the yaw primarily, such that the AUV will always be subject to an uncontrollable yaw moment, resulting in the failure of path following. Therefore, it is necessary to carry out the fault detection of the propellers independently [10].

When the motion state of an AUV is represented by the state equation, we can utilize the observations to estimate the unknown state variables. Therefore, a model-based fault diagnosis method was considered: based on the states observed, we could detect the fault and estimate the fault degree [11]. Fault diagnosis based on the analytic model is the oldest and most systematic method; for example, Matko [12] applied the sigma-point unscented Kalman filter method to two types of AUVs in an open-water navigation task simulation, and Filaretov et al. [13] solved the problem of fault detection and localization using the kinematic model of the AUV, with special data fusion from its navigation sensors. Chu et al. [14] presented a propeller fault reconstruction method based on a terminal sliding observer. Sun et al. [15] proposed the fault diagnosis method based on a Gaussian particle filter, introducing the yaw moment loss parameter to estimate the fault. The idea of using the modified Bayesian algorithm to detect faults is worth learning from; however, as only a certain degree of freedom in the control force and yaw moment losses is used, the method may misdiagnose fault-free situations. In addition, because the method lacks forecasts for future trends, the fault is detected relatively late.

According to the principle of rank statistics [16], we can conclude that the sampling points obtained by the rank sampling method are reasonable and can simulate the probability distribution of the system state effectively. Further, the particle filtering method has high filtering precision, and can track system state changes with time. In the literature [17], to solve the problem of particle diversity and effectiveness lost due to particle degeneracy and resampling, the rank filter and particle filter methods were combined. First, the importance density function was obtained by the rank filtering method. As the importance density function contains the latest observation information, it is closer to the true state probability density; the particle filter method is used subsequently to estimate the system state. This allows the rank particle filter to have high filtering accuracy, while eliminating the complexity of calculations in the particle resampling method.

In view of the theories above, this study applied the rank particle filter method to the fault diagnosis technology. First, the mathematical model of the AUV was modelled by hydrodynamic parameters. The probability density function of the motion state of the AUV was obtained by the rank filtering method, and its trend was predicted at this time. Subsequently, the particle filter was combined to construct the rank particle filter, and the 
control force and yaw moment losses in the AUV were estimated. Further, the modified Bayes (MB) algorithm was used to analyse the estimated losses over a time period. Finally, the back propagation (BP) neural network was used for propeller fault diagnosis. With the simulation data and the sea trail data with the propeller fault, the fault diagnosis simulation experiments were carried out to test the performance of the proposed method.

\section{AUV Mathematical Model and Fault Model}

\subsection{AUV Platform and Hydrodynamic Model}

Because the main target of the fault diagnosis is the propeller of the AUV, a certain type of AUV was selected as the research object. Propellers were the only actuators of this AUV, and no fin was used in its motion control.

To simplify the complexity of the fault diagnosis algorithm, the mathematical model of the AUV system was decomposed in the horizontal and vertical planes. Only the planar motion of the AUV was observed, and it was assumed that the fault occurred in the two stern propellers in the horizontal plane. The AUV stern propellers' configuration in the horizontal plane is shown in Fig. 1. The efficiency of the channel propellers will reduce at high velocities, therefore the turning motion is primarily controlled by these two stern propellers. The two propellers adopt catheter propellers, in which the longitudinal axis of each propeller and that of the AUV form a $13^{\circ}$ angle. The main parameters of the AUV are listed in Table 1.

Table 1 Main hydrodynamic parameters of the AUV

\begin{tabular}{|c|l||l|l|}
\hline $\mathrm{m}$ & $2.15 \times 10^{-3} \mathrm{~kg}$ & $\mathrm{~L}$ & $5.6 \mathrm{~m}$ \\
\hline $\mathrm{b}$ & $1 \mathrm{~m}$ & $\mathrm{~h}$ & $1 \mathrm{~m}$ \\
\hline $\mathrm{G}$ & $(0.09,0,-0.022) \mathrm{m}$ & $I_{\mathrm{x}}$ & $5.42 \times 10^{2} \mathrm{~kg}$ \\
\hline$I_{y}$ & $7.58 \times 10^{3} \mathrm{~kg}$ & $I_{z}$ & $7.62 \times 10^{3} \mathrm{~kg}$ \\
\hline$\dot{X}_{\dot{u}}^{\prime}$ & $-1.58 \times 10^{-3}$ & $\dot{Y}_{\dot{v}}^{\prime}$ & $-8.154 \times 10^{-2}$ \\
\hline$\dot{Z}_{\dot{w}}^{\prime}$ & $-7.63 \times 10^{-2}$ & $\dot{N}_{\dot{v}}^{\prime}$ & $-3.88 \times 10^{-3}$ \\
\hline$\dot{Y}_{\dot{r}}^{\prime}$ & $-8.09 \times 10^{-3}$ & $\dot{M}_{\dot{w}}^{\prime}$ & $3.80 \times 10^{-3}$ \\
\hline
\end{tabular}

The established space coordinate system is defined as recommended by the International Ship Model Towel Pool Recall (ITTC) and the Shipbuilding and Engineering Society (SNAME) terminology bulletin system, as shown in Fig. 2.

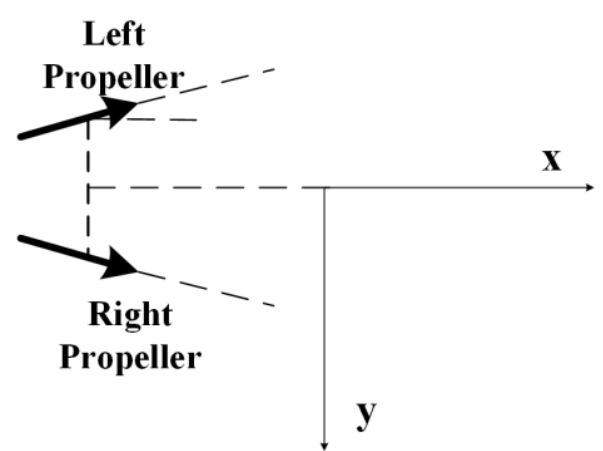

Fig. 1 Propeller configuration in horizontal plane

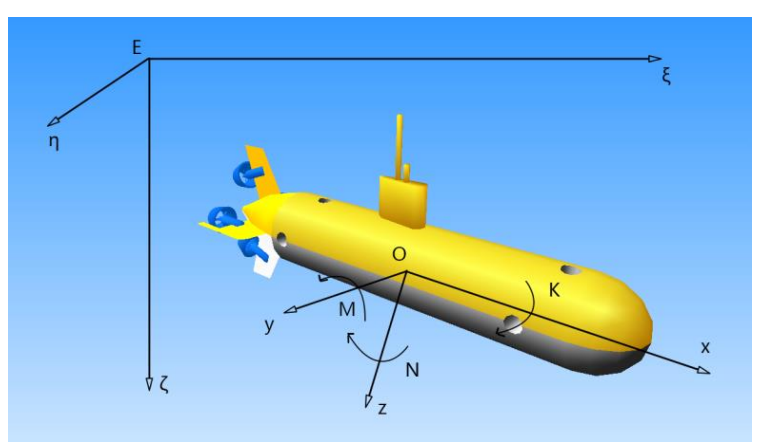

Fig. 2 AUV space coordinate system

The $E-\xi \eta \zeta$ geodetic coordinate system is fixed on the earth at any fixed point, and the origin of the body coordinate system $O-x y z$ is fixed at the AUV's centre of gravity. 
Furthermore, the geodetic coordinate system and the body system can reach the corresponding relations through the conversion:

$$
\left[\begin{array}{l}
x \\
y \\
z
\end{array}\right]=\mathbf{T}^{-1}\left[\begin{array}{c}
\xi \\
\eta \\
\zeta
\end{array}\right]
$$

In this equation,

$$
\mathbf{T}^{-1}=\left[\begin{array}{ccc}
\cos \psi \cos \theta & \sin \psi \cos \theta & -\sin \theta \\
\cos \psi \sin \theta \sin \phi-\sin \psi \cos \phi & \sin \psi \sin \theta \sin \phi+\cos \psi \cos \phi & \cos \theta \sin \phi \\
\cos \psi \sin \theta \cos \phi+\sin \psi \sin \phi & \sin \psi \sin \theta \cos \phi-\cos \psi \sin \phi & \cos \theta \cos \phi
\end{array}\right]
$$

where $\varphi$ is the roll angle, and the AUV tilting to the right is defined as positive; $\theta$ is the pitch angle, and the AUV tilting to the stern is defined as positive; $\psi$ is the yaw angle, and the AUV turning to the right is defined as positive.

We assumed that the AUV operated in a deep and still environment; thus, the control force was produced by the main body hydrodynamic force and the propeller thrust, according to equation (3):

$$
F=f(V, \dot{V}, \Omega, \dot{\Omega}, n)
$$

where $V$ and $\dot{V}$ are the velocity and acceleration of the AUV, respectively; $\Omega$ and $\dot{\Omega}$ are the angular velocity and angular acceleration of the AUV, respectively; $n$ is the propeller's rotating speed.

Through a Taylor expansion at the reference point, the hydrodynamic coefficients in the formula above were obtained, while ignoring the second-order coefficients above. Based on the previous assumptions, the inertial hydrodynamic terms were attributed to the terms on the left side of the equation, and the non-inertial hydrodynamic terms were attributed to the terms on the right side of the equation. By setting $\mathbf{X}=\left[\begin{array}{llllll}u & v & w & p & q & r\end{array}\right]^{\prime}$, we obtained

$$
D \dot{X}=F_{\text {vis }}+F_{\text {propeller }}
$$

In the equation,

$$
\begin{gathered}
D=\left[\begin{array}{cccccc}
m-X_{\dot{u}} & -X_{\dot{v}} & -X_{\dot{w}} & 0 & m z_{G}-X_{\dot{q}} & -X_{\dot{r}} \\
-Y_{\dot{u}} & m-Y_{\dot{v}} & 0 & -m z_{G}-Y_{\dot{p}} & 0 & m x_{G}-Y_{\dot{r}} \\
-Z_{\dot{u}} & 0 & m-Z_{\dot{w}} & -Z_{\dot{p}} & -m x_{G}-Z_{\dot{q}} & 0 \\
0 & -m z_{G}-K_{\dot{v}} & -K_{\dot{w}} & I_{x}-K_{\dot{p}} & -K_{\dot{q}} & -K_{\dot{r}} \\
m z_{G}-M_{\dot{u}} & 0 & -m x_{G}-M_{\dot{w}} & -M_{\dot{p}} & I_{y}-M_{\dot{q}} & 0 \\
-N_{\dot{u}} & m x_{G}-N_{\dot{v}} & 0 & -N_{\dot{p}} & 0 & I_{z}-N_{\dot{r}}
\end{array}\right] \\
F_{v i s}=\left[\begin{array}{llllll}
X_{v i s} & Y_{v i s} & Z_{v i s} & K_{v i s} & M_{v i s} & N_{v i s}
\end{array}\right]
\end{gathered}
$$

$F_{v i s}$ is the non-inertial hydrodynamic term; $F_{\text {propeller }}$ is the thrust generated by the propeller.

The motion system of the AUV is highly nonlinear and can be described by the following equation of state: 


$$
\left\{\begin{array}{l}
\dot{x}=A x+B u \\
\text { Zobser }=C x,
\end{array}\right.
$$

where $A$ is the system matrix, $x$ is the vector of the motion state variables, $u$ is the control input, $B$ is the input matrix of the system, Zobser is the measured value, and $C$ is the observation matrix of the system.

\subsection{AUV Platform Fault Model}

In Section 2.1, the state equation of the AUV was obtained, which was a continuous equation. However, in the actual operation, the status of the AUV is periodically sampled. Similarly, the corresponding control command is also periodically sent to the propellers. Therefore, the discretization for the continuous equation above is needed:

$$
\left\{\begin{array}{l}
x_{k+1}=G_{k} \cdot x_{k}+H_{k} \cdot u_{k}+Q_{k} \\
\text { Zobser }_{k+1}=C_{k} \cdot x_{k}+R_{k},
\end{array}\right.
$$

where $G_{k}$ is the discretized system matrix, $H_{k}$ is the discretized input matrix of the system, $Q_{k}$ and $R_{k}$ are the system process noise and measurement noise, respectively.

The failure in the propeller will weaken the output thrust; therefore, the actual axial control force and the yaw moment of the AUV will change. The actual control force and yaw moment will deviate from $u_{k}$ to $u_{k}+\Delta u_{k}$, where $\Delta u_{k}$ in the normal state should be zero. To diagnose the AUV's state, the actual control force and yaw moment, which include the fault condition are introduced into equation (6), and the motion model including the failure of the AUV can be obtained as shown in equation (7):

$$
\left\{\begin{array}{l}
x_{k+1}=G_{k} \cdot x_{k}+H_{k} \cdot\left(u_{k}+\Delta u_{k}\right)+Q_{k} \\
\text { Zobser }_{k+1}=C_{k} \cdot x_{k}+R_{k}
\end{array}\right.
$$

\section{Rank Particle Filtering and Improved Algorithm}

\subsection{Rank Filtering Method}

The rank sampling method is a deterministic sampling method. First, we determined the state vector $X$ with a mean value $\bar{X}$ and the covariance matrix $\operatorname{Cov}_{x}$, and then calculated the mean and mean square error of the nonlinear change $f(X)$.

According to $\bar{X}$ and $\operatorname{Cov}_{x}$, we obtained the initial sample points xSigmaPts : the $i^{\text {th }}$ sample point was

$$
x \operatorname{SigmaPts}(i)=\bar{X}+\mu_{j} \lambda_{p j}\left(\sqrt{\operatorname{Cov}_{X}}\right)_{l}
$$

In this case, $l=1,2, \ldots, R_{n}\left(R_{n}\right.$ is the dimension of $\left.X\right)$, and when $j=1,2, \ldots, R_{m}\left(R_{m}\right.$ represents the layers of rank sampling, and the more the layers, the higher is the precision of the sampling), $i=(j-1) R_{n}+1 . \lambda_{p j}$ is the lower bound of the confidence interval with probability $p_{j}$ in the $X$ distribution. $\mu_{j}$ is the correction factor of the sampling point, subjected to 


$$
\sum_{j=1, j \neq R_{m}+1}^{2 R_{m}+1} \mu_{j} \lambda_{p j}=0
$$

The initial sampling points were subject to non-linear changes to obtain the set of output variables $x$ Pred:

$$
x \operatorname{Pred}(i)=f(x \operatorname{SigmaPts}(i))
$$

Next, the mean and covariance were calculated:

$$
\begin{gathered}
\overline{x P r e d}=\frac{1}{n P t s} \sum_{i=1}^{n P t s} x \operatorname{Pred}(i) \\
\text { Cov }_{x P r e d}=\frac{1}{\omega} \sum_{i=1}^{n P t s} \mu_{i}(x \operatorname{Pred}(i)-\overline{x P r e d})(x \operatorname{Pred}(i)-\overline{x P r e d})^{\mathrm{T}}
\end{gathered}
$$

Here, the weight coefficient of the covariance is $\omega$

$$
\omega=\sum_{j=1, j \neq R_{m}+1}^{2 R_{m}+1} \mu_{j}^{2} \lambda_{p j}^{2}
$$

\subsection{Rank Particle Filter with Multi-Step-Ahead Prediction}

The rank particle filter method was used subsequently to estimate the future state of the AUV. The importance density function was obtained by the rank filtering method in Section 3.1 above, and then the particle filter method was combined to estimate the future state.

We knew that $x$ SigmaPts was the initial sample points from the posterior probability distribution $\mathrm{N}\left(\bar{X}, \operatorname{Cov}_{x}\right)$, and it was updated to generate the $x$ Pred and corresponding weights via the rank filter method above. Further, we assumed that $x \operatorname{Pred}(k, \mathrm{i})$ was one of the sample points in $x$ Pred $(k)$ at time $\mathrm{k}$ obtained by the particle filter sampling process.

We defined a discrete distribution that contained the sample points and weights $\omega(k, i)$ :

$$
\hat{p}\left(x \operatorname{Pred}(k) \mid \text { Zobser }_{0: k}\right)=\sum_{i=1}^{N} \omega(k, i) \delta(x \operatorname{Pred}(k)-x \operatorname{Pred}(k \mid k-1, i))
$$

where $\delta(x)$ is the Dirac delta function,

and the p-step-ahead prediction probability density function could be obtained based on the following distribution:

$$
p\left(x \operatorname{Pred}(k+p) \mid \operatorname{Zobser}_{0: k}\right)=\int p\left(x \operatorname{Pred}(k) \mid \operatorname{Zobser}_{0: k}\right) \cdot\left[\prod_{j=k+1}^{k+p} p(x \operatorname{Pred}(j) \mid x \operatorname{Pred}(j-1)] d x_{k: k+p-1}\right.
$$

Subsequently, $p\left(x \operatorname{Pred}(k) \mid\right.$ Zobser $\left._{0: k}\right)$ in this equation was an alternative by the particles obtained by the rank sampling method:

$$
\sum_{i=1}^{N} \omega(k, i) \int p(x \operatorname{Pred}(k+1) \mid x \operatorname{Pred}(k, i)) \cdot \prod_{j=k+2}^{k+p} p(x \operatorname{Pred}(j) \mid x \operatorname{Pred}(j-1)) d x_{k+1: k+p-1}
$$


If $p \geq 2 \in \mathrm{N}^{*}$, by calculating the integrals, $x \operatorname{Pred}(k, \mathrm{i})$ in $x \operatorname{Pred}(k)$ could now be extended. The $\mathrm{p}$-step prediction algorithm for the rank particle filter was obtained as follows:

For $j=1,2, \ldots, \mathrm{p}$

For $i=1,2, \ldots, \mathrm{N}$

sample $x \operatorname{Pred}(k+j \mid k+j-1, i)$ from $p(x \operatorname{Pred}(k+j) \mid x \operatorname{Pred}(k+j-1))$

end

end

The multi-step-ahead prediction probability density function was then obtained, and the weights $\omega(k, i)$ were maintained as those at time $\mathrm{k}$ :

$$
\hat{p}\left(x \operatorname{Pred}(k+p) \mid \text { Zobser }_{0: k}\right)=\sum_{i=1}^{N} \omega(k, i) \delta(x \operatorname{Pred}(k+p)-x \operatorname{Pred}(k+p \mid k+p-1, i))
$$

Finally, we could resample the prediction particles from this distribution and repeated the prediction process in the next iteration.

\section{AUV Propeller Fault Diagnosis Method}

\subsection{Constructing the Rank Particle Filter}

Using the fault diagnosis model obtained in Section 2.2, the following rank particle filter was constructed:

$$
\left\{\begin{array}{l}
\hat{x}_{k+1}=G_{k} \cdot \hat{x}_{k}+H_{k} \cdot\left(u_{k}+\Delta u_{k}\right)+Q_{k} \\
\hat{Z}_{\text {obser }}+1=C_{k} \cdot \hat{x}_{k}+R_{k}
\end{array}\right.
$$

The goal of the fault diagnosis is to analyse the value $\Delta u_{k}$ and its trend, of which the mean value should be zero in the normal state. When the propeller fails, the thrust loss and additional yaw moment lead to changes in $\Delta u_{k}$. The amplitude of the changes is determined by the gap between the desired and the actual control force and yaw moment. Therefore, we considered $\Delta u_{k}=\{\Delta X, \Delta Y, \Delta Z, \Delta K, \Delta M, \Delta N\}$ as the estimated term of the AUV state.

\subsection{Fault Diagnosis Method}

The MB algorithm was then used to analyse the time series of the AUV states. The mean value $\Delta \overline{\hat{u}}_{k}$, the variance $S_{2}(k)$, the relative normal mean value $\Delta \bar{u}_{k}$, and the variance $S_{1}(k)$ of the N-point state estimation values were obtained by the following operations, based on the $\Delta \hat{u}_{k}$ estimated at $\mathrm{N}$ time points respectively before time $\mathrm{k}$ :

$$
\begin{aligned}
& \Delta \overline{\hat{u}}_{k}(k)=\frac{1}{N} \sum_{j=1}^{N} \Delta \hat{u}_{k}(k-j) \\
& S_{1}(k)=\frac{1}{N-1} \sum_{j=1}^{N}\left[\Delta \hat{u}_{k}(k-j)-\Delta \bar{u}_{k}\right]^{2}
\end{aligned}
$$




$$
\begin{aligned}
& S_{2}(k)=\frac{1}{N-1} \sum_{j=1}^{N}\left[\Delta \hat{u}_{k}(k-j)-\Delta \overline{\hat{u}}_{k}(k)\right]^{2} \\
& M B(k)=\frac{S_{1}(k)}{Q^{\prime}}-\ln \left(\frac{S_{2}(k)}{Q^{\prime}}\right)-1
\end{aligned}
$$

If the fault was diagnosed directly by the MB value $M B(k)$ above using the threshold method, owing to the use of only one degree of freedom, problems would occur. For example, when considering the state-estimated value in the $\mathrm{X}$-axis direction alone, thrust losses would also exist except in fault conditions, as a result of the accelerating process or the presence of ocean current interference. This might lead to the misdiagnosis of a normal condition; therefore, a BP neural network algorithm was combined, considering its pattern recognition ability.

As shown in Fig. 3, the control force and yaw moment losses, and the MB values were taken as the input, and the output was the diagnostic value indicating whether the fault existed. The thrust loss and diagnostic values in normal conditions were selected as training samples to train the neural network.

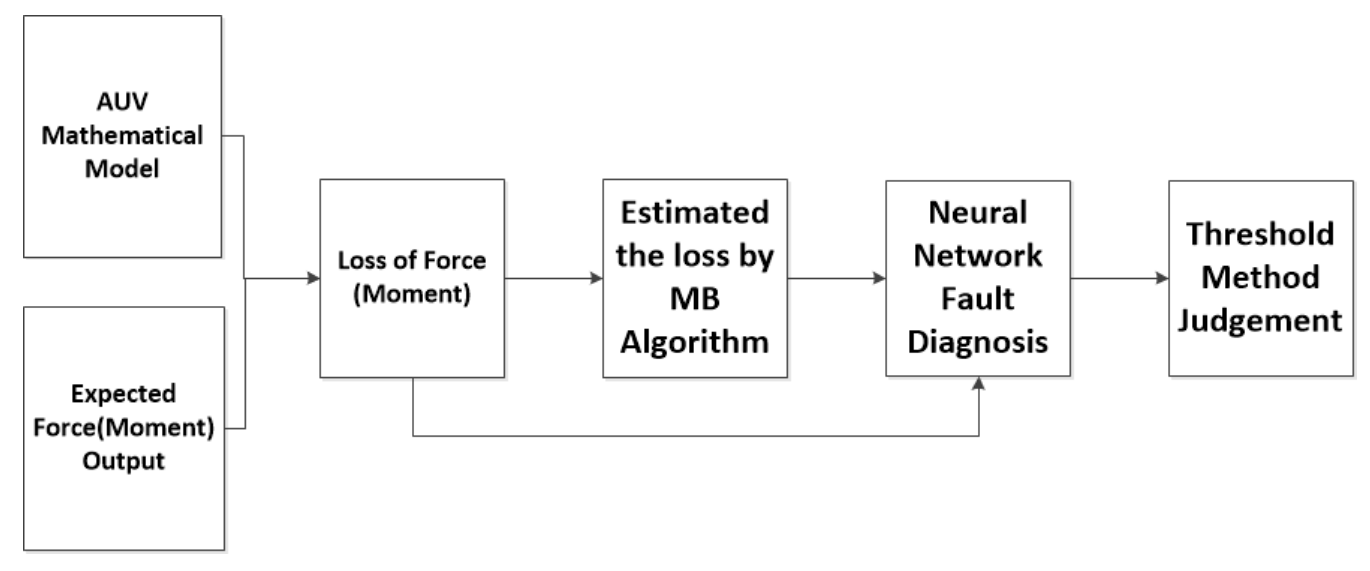

Fig. 3 Fault diagnosis algorithm process

\subsection{Rank Particle Filter Propeller Fault Diagnosis Method}

Based on the theories above, the fault diagnosis process is described below:

Step 1: Initialize the system state values, and give the initial state $x_{0}=\{u, v, r, \Delta X, \Delta Y, \Delta N\} \quad$ first; subsequently, obtain the particle set $\{x \operatorname{SigmaPts}(i), 1 / N\}(i=1,2 \ldots, N)$ from the initial distribution.

Step 2: Obtain a new set of particles $\{\hat{x} \operatorname{PredSigmaPts}(k, i)\}(i=1,2 \ldots, N)$ by the rank sampling method.

Step 3: Use the rank particle filter method to obtain the posterior probability density function, which is then considered to be the proposed distribution

Step 4: Use the multi-step-ahead prediction method, described in Section 3.2, to update the sample distribution.

Step 5: Obtain the new particle set $\{x \operatorname{SigmaPts}(k, i)\}(i=1,2 \ldots, N)$ from the proposed distribution. The important weights are taken as the likelihood probability density function, and are normalized using the weighted set of particles, such that the mean and covariance of the probability distribution at $\mathrm{k}$ can be calculated. 
Step 6: Estimate the AUV state $\Delta \hat{u}$ based on the particle set and the normalized weights, and use the MB algorithm to manage $\Delta \hat{u}$.

Step 7: Take the AUV state estimation $\Delta \hat{u}$, and the MB value obtained by the MB algorithm as the input to the BP neural network. If the diagnostic value is beyond a certain value, a fault is considered to have occurred.

\section{Fault Diagnosis Simulation}

\subsection{Simulation Environment Configuration}

To simplify the complexity of the fault diagnosis algorithm, the mathematical model of the AUV system was decomposed in the horizontal and vertical planes as described in Section 2. Assuming that one of the stern propellers in the horizontal plane was faulty, the fault only affects three degrees of freedom in the horizontal plane. Thus, we primarily discuss the fault situation simulation in the horizontal plane, and use the fault diagnosis algorithm in this paper to diagnose the fault.

During the actual sea trail with this AUV, we found that a faulty propeller might not output enough thrust to meet the desired thrust, owing to the fault in the blade. In addition, another fault state existed: when the desired thrust was small, the propeller could output the required thrust; however, when the desired thrust was greater than a certain value, the propeller could not output the desired output thrust, instead, a lower thrust was output.

Therefore, the failure condition of the AUV's left stern propeller was set as follows: when the output thrust of the propeller was less than $60 \mathrm{~N}$, the propeller was considered to be rotating at a low speed, and the thrust of the propeller could be output normally; when the desired thrust of the propeller was greater than $60 \mathrm{~N}$, this faulty propeller could only output $60 \mathrm{~N}$ thrust. In this case, the fault diagnosis result was analysed. Considering that the desired output thrust of the propeller was related to the desired velocity and yaw angle of the AUV, the simulation parameters were set based the different desired velocities and yaw angles of the AUV.

The accelerating process, or the turning of the bow during acceleration will cause thrust loss, which may be misdiagnosed by the algorithm as a fault. In determining the threshold, we carried out a set of simulations under different desired velocities and yaw angles, where no fault occurred in the propeller model, and obtained the MB values caused by the motion changes in the AUV. In Table 2, the maximum MB values in fault-free conditions, diagnosed by the MB algorithm under different AUV motion states, are enumerated:

Table 2 MB values in fault-free conditions

\begin{tabular}{|l|l|l|l|}
\hline AUV Status & X-axis & Y-axis & Z-axis \\
\hline Accelerate & 26 & 8 & 8 \\
\hline Accelerate (Turn) & 13 & 14 & 155 \\
\hline Turn & 9 & 8 & 8 \\
\hline Cruise & 9 & 8 & 8 \\
\hline
\end{tabular}

The maximum operating velocity of the AUV in the simulation was $2.0 \mathrm{~m} / \mathrm{s}$; therefore, a series of simulations were carried out, where the desired velocities range from 0 to $2.0 \mathrm{~m} / \mathrm{s}$ and different yaw angles were selected. The MB values of the AUV in different motion states characterized the AUV states during a time period. The threshold should be set to improve the sensitivity of the algorithm, and provide the basis for the comparison of different algorithms. 
We considered that if the MB value of the fault diagnosis algorithm was greater than the maximum $\mathrm{MB}$ value at a certain degree of freedom in a fault-free state, the propeller was considered to be possibly faulty. In this case, the maximum MB values obtained with different degrees of freedom respectively in Table 2 were set as the threshold.

\subsection{Simulation and Results Analysis}

First, the case of an AUV cruising in a straight line was simulated: the desired velocity was set as $0.5 \mathrm{~m} / \mathrm{s}$ during the first $50 \mathrm{~s}$; from $50 \mathrm{~s}$ to $100 \mathrm{~s}$, and the desired velocity was 1.0 $\mathrm{m} / \mathrm{s}$. The desired velocity of the AUV was set as $2.0 \mathrm{~m} / \mathrm{s}$ starting from $100 \mathrm{~s}$.

Figures 4-6 show the simulation results, describing the use of the rank particle filter method. The control command and the current motion state of the AUV were taken as the input, and the control force and yaw moment losses and their multi-step-ahead predictions in different degree of freedom were shown. The solid line is the estimated result, and the dashdot line is the result of the forward prediction. The results show that the multi-step-ahead prediction will amplify the original control force and yaw moment losses.
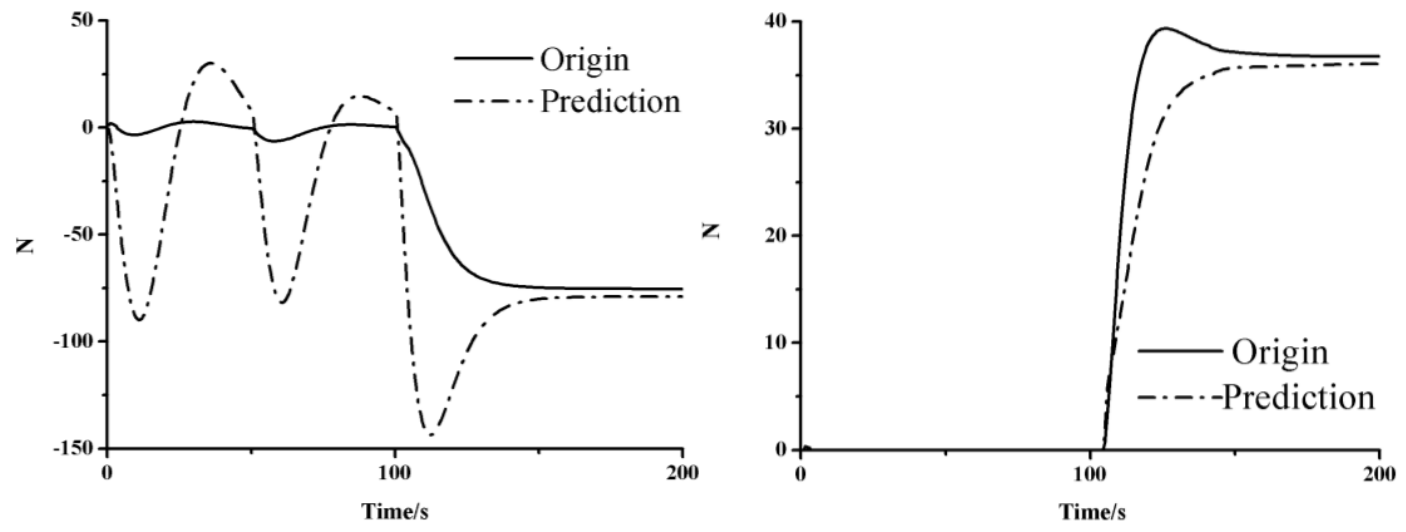

Fig. $4 \mathrm{X}$-axis force loss estimation and prediction Fig. 5 Y-axis force loss estimation and prediction

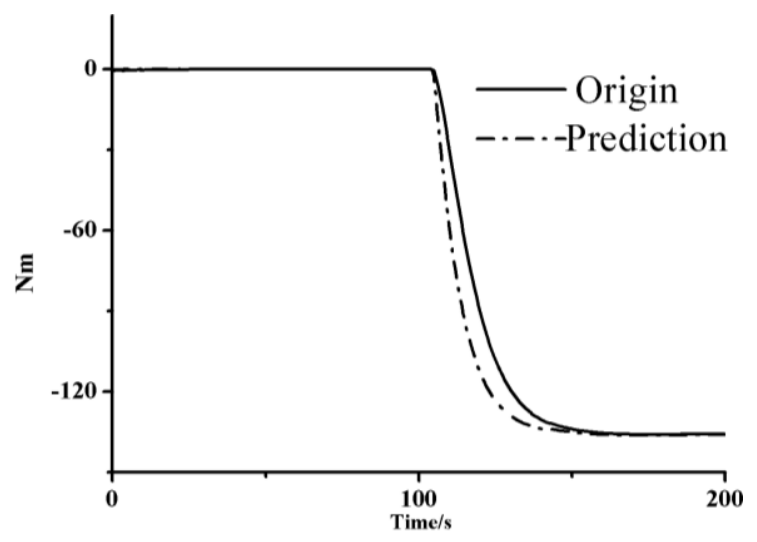

Fig. 6 Yaw moment loss estimation and prediction

Based on the MB algorithm, the control force and yaw moment losses are used to calculate the trend in a certain time period using the sliding time window, and the threshold method is used to judge whether a fault had occurred. Figures $7-10$ show the results. Fig. 7 shows that the calculation result in the $\mathrm{X}$-axis direction is greater than the set threshold value of 26 at $110.5 \mathrm{~s}$, and the fault diagnosis algorithm considers that a fault has occurred at this time. 


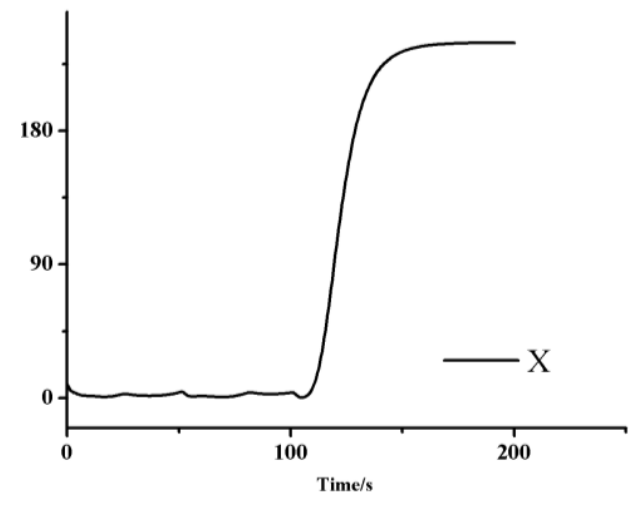

Fig. $7 \mathrm{X}$-axis force loss with MB algorithm

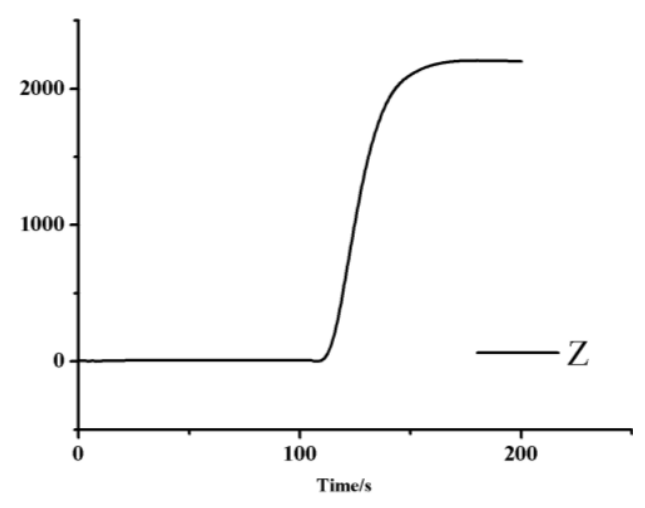

Fig. 9 Yaw moment loss with MB algorithm

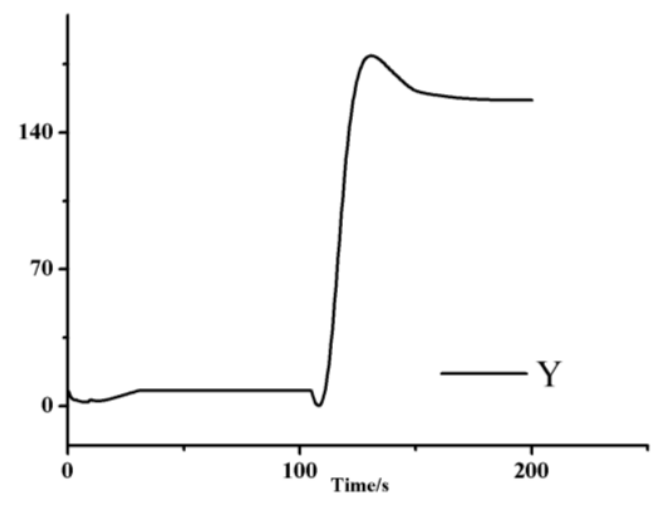

Fig. 8 Y-axis force loss with MB algorithm

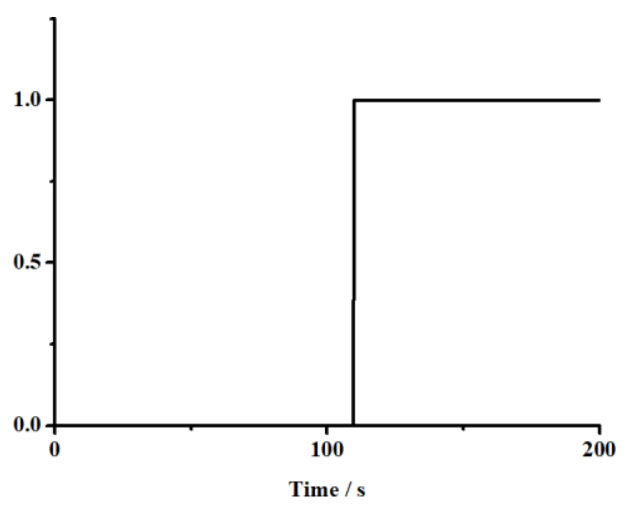

Fig. 10 Threshold method diagnosis result

The control force and the yaw moment losses prediction were also analysed by the MB algorithm. The threshold method was also used to judge whether a fault has occurred. Figure 14 shows that the fault diagnosis algorithm with prediction diagnosed the potential failure earlier, at $105 \mathrm{~s}$. However, between $7 \mathrm{~s}$ to $23 \mathrm{~s}$ and between $57 \mathrm{~s}$ to $73 \mathrm{~s}$, the AUV was accelerating and the thrust loss in the X-axis direction was amplified by the multi-step-ahead prediction, resulting in a larger estimated thrust loss. Although the MB values in the Y-axis and Z-axis directions showed that the AUV was in a normal state, a fault was error detected, owing to the $\mathrm{X}$-axis value that is beyond the threshold set previously. Therefore, it would not be accurate if only the loss in a single degree of freedom is considered to determine the fault.

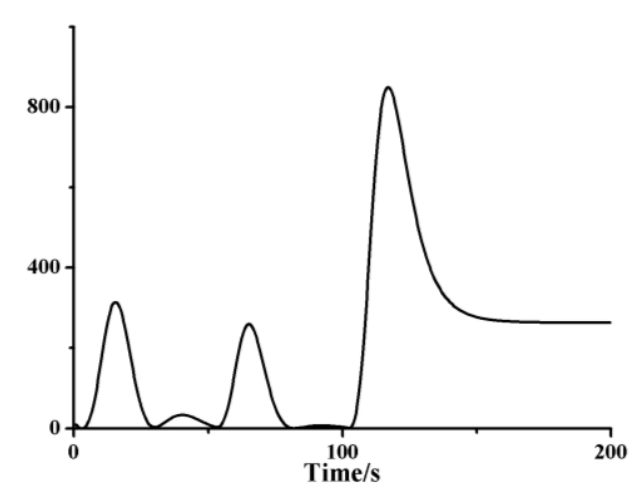

Fig. $11 \mathrm{X}$-axis force loss prediction with MB

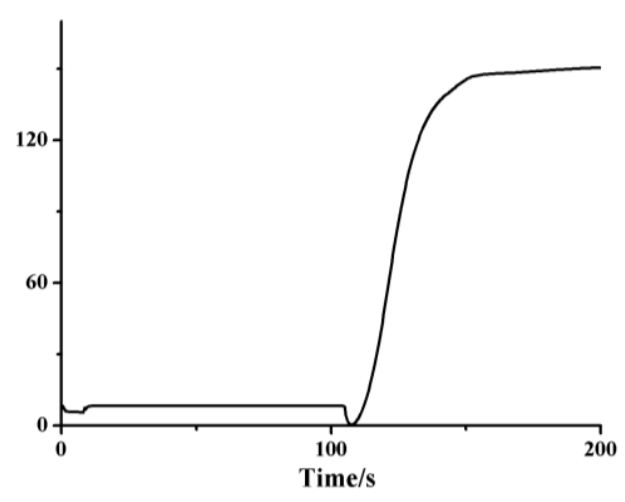

Fig. 12 Y-axis force loss prediction with MB 


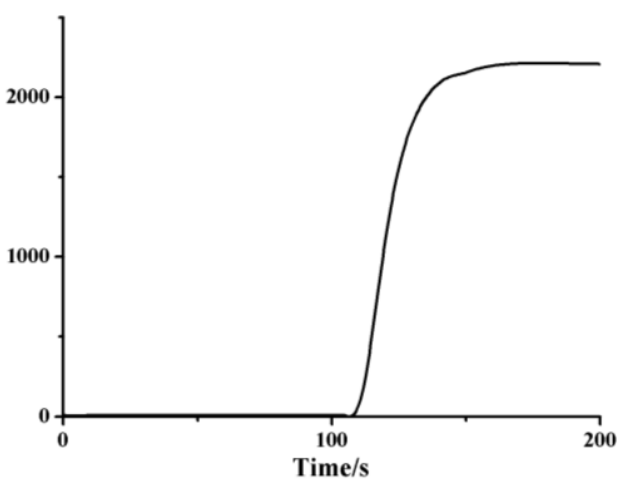

Fig. 13 Yaw moment loss prediction with MB

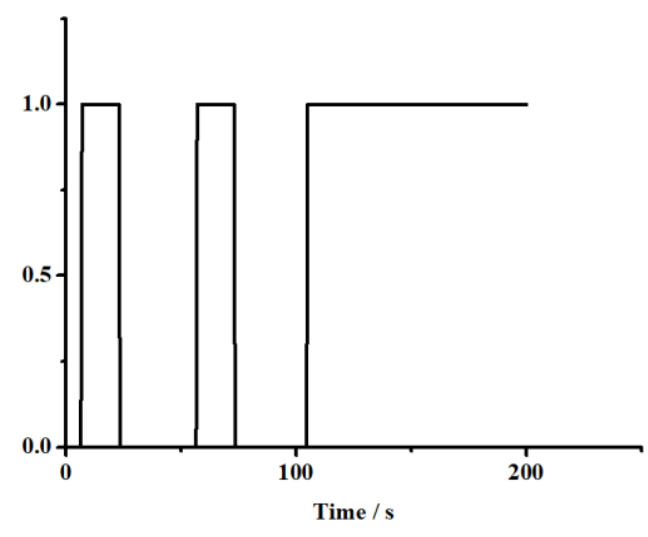

Fig. 14 Threshold method diagnosis result

The comparison above shows that the control force and yaw force losses prediction can reflect the trend, and can detect an imminent fault sooner. However, only a certain degree of freedom of loss was used, and was not enough to eliminate the misdiagnosis. Considering the propeller fault configuration again: the left main propeller could output a thrust below $60 \mathrm{~N}$ normally, but could not provide enough thrust beyond $60 \mathrm{~N}$. When the AUV was no longer at a standstill, a flow had caused resistance during acceleration. Furthermore, a delay response occurred between the control command and the execution of the actuator in the actual sea trail. This caused the estimated thrust output to deviate from the expected thrust. This deviation was detected, and also amplified in the multi-step-ahead prediction process, which resulted in an increase of the MB values. Faults were error determined to occur.

Therefore, the control force and yaw moment losses with only one degree of freedom should not be analysed. All the control force losses in the X-axis and Y-axis, the yaw moment in the Z-axis, and the corresponding MB values should be taken into account. Subsequently, the normalized values were used as the input of the BP neural network, which was trained by the values in the normal state, and the fault situation could be diagnosed.

The BP neural network is capable of pattern recognition, and we expected the diagnostic value to be approximately zero in the normal states. During the training process, the control forces and the yaw moments, the MB values, and the expected diagnostic values in the normal states were taken as samples. According to the previous simulations, a thrust loss due to acceleration was estimated. Thus, we expected that when the AUV was accelerating when cruising in a straight line or when turning the bow, the output should be 0.2 and 0.3 , respectively. Some examples for training the neural network are given in Table 3.

The state vectors included the control force, yaw moment losses, and the MB values; the BP neural network was trained by those values. Under normal circumstances, the diagnostic value of the neural network is up to 0.3 . If the diagnostic value was close to 0 , it indicated that the AUV was in a normal state. However, if the diagnostic value was farther away from 0 , it indicated that the AUV might be faulty. In this study, the system was considered to be faulty if the diagnostic value was greater than 0.3 .

Table 3 BP neural network training samples

\begin{tabular}{|l|l|l|}
\hline AUV Status & \multicolumn{1}{|c|}{ Example of Samples } & Expected Output \\
\hline Accelerate & {$[-4.35,0.04,0.13,-87.55,0,0]$} & 0.2 \\
\hline Accelerate (Turn) & {$[3.26,5.54,7.84,1.44,2.1,11.02]$} & 0.3 \\
\hline Turn & {$[-75.3,36.7,-135,-79.3,35.9,-136.2]$} & 0 \\
\hline Cruise & {$[0.68,0,0,-3.76,0,0]$} & 0 \\
\hline
\end{tabular}


As shown in Fig. 15, when the diagnostic value deviates from 0 during the previous two accelerating processes, the output value of the fault diagnosis result is always below the threshold value of 0.3. At $108 \mathrm{~s}$, the diagnostic value of the BP neural network is greater than the threshold value of 0.3 for the first time, which means that the AUV experienced an imminent fault. The comparison between Fig. 15 and Fig. 14 show that the fault diagnosis using the BP neural network can avoid the misdiagnosis when the AUV is accelerating. Compared with the fault diagnosis results obtained by the fault diagnosis algorithm without prediction in Fig. 10, we observed that the original method detected the fault in the propeller at $110.5 \mathrm{~s}$, and the proposed method showed that the AUV was faulty at $108 \mathrm{~s}$. The proposed method can detect the AUV failure at the soonest possible.

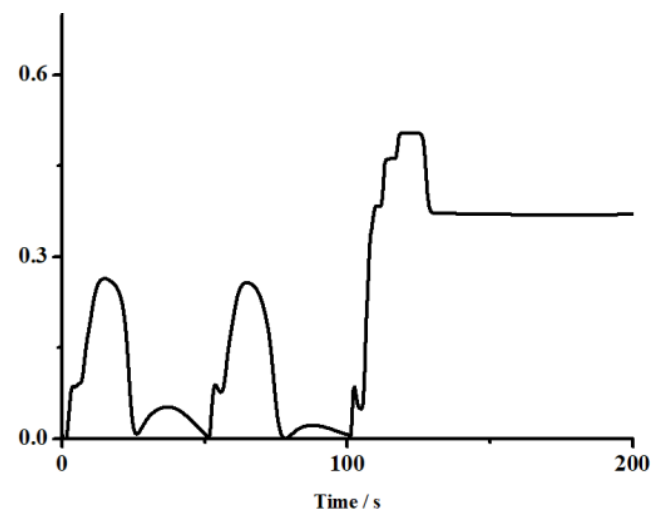

Fig. 15 BP neural network diagnosis result

Next, the case of an AUV turning the bow during acceleration was simulated: from $0 \mathrm{~s}$ to $100 \mathrm{~s}$, the AUV was released and accelerated to $0.5 \mathrm{~m} / \mathrm{s}$; from $100 \mathrm{~s}$ to $200 \mathrm{~s}$, the AUV was turning $45^{\circ}$ to the left; subsequently, the AUV was accelerating from $0.5 \mathrm{~m} / \mathrm{s}$ to $1.0 \mathrm{~m} / \mathrm{s}$ while turning its bow $90^{\circ}$ to the left starting from $200 \mathrm{~s}$. Fault diagnosis algorithms with and without prediction were applied to judge whether a fault has occurred.

Figures 16-19 show the simulation results. Fig. 17 shows that the MB value in the Zaxis was greater than the set threshold value of 155 at $212 \mathrm{~s}$, and the fault diagnosis algorithm considered that a fault had occurred at this time. In addition, from Fig. 18 and Fig. 19, we could conclude that the diagnosis algorithm with prediction detected the fault sooner, and the diagnostic value of the BP neural network exceeded the threshold at $202.5 \mathrm{~s}$.

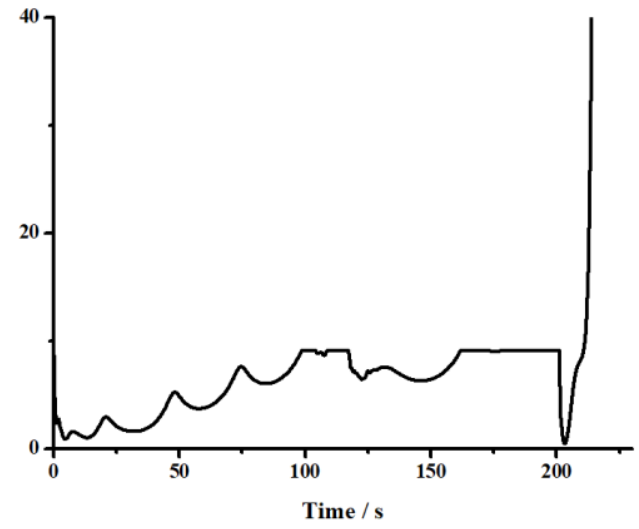

Fig. $16 \mathrm{X}$-axis force loss with $\mathrm{MB}$ algorithm

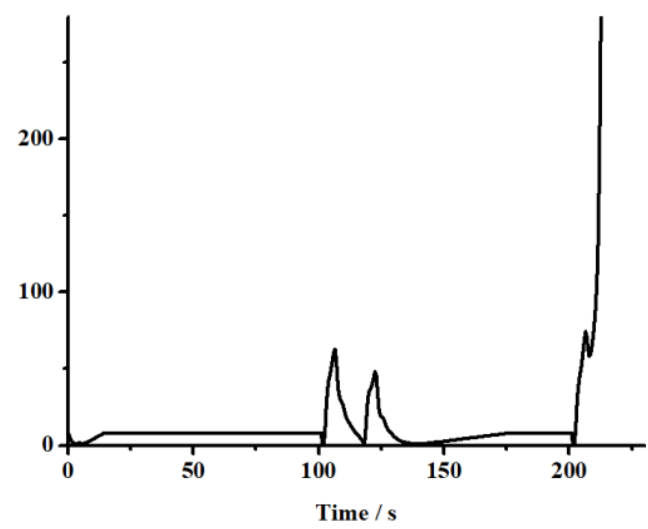

Fig. 17 Yaw moment loss with MB algorithm 

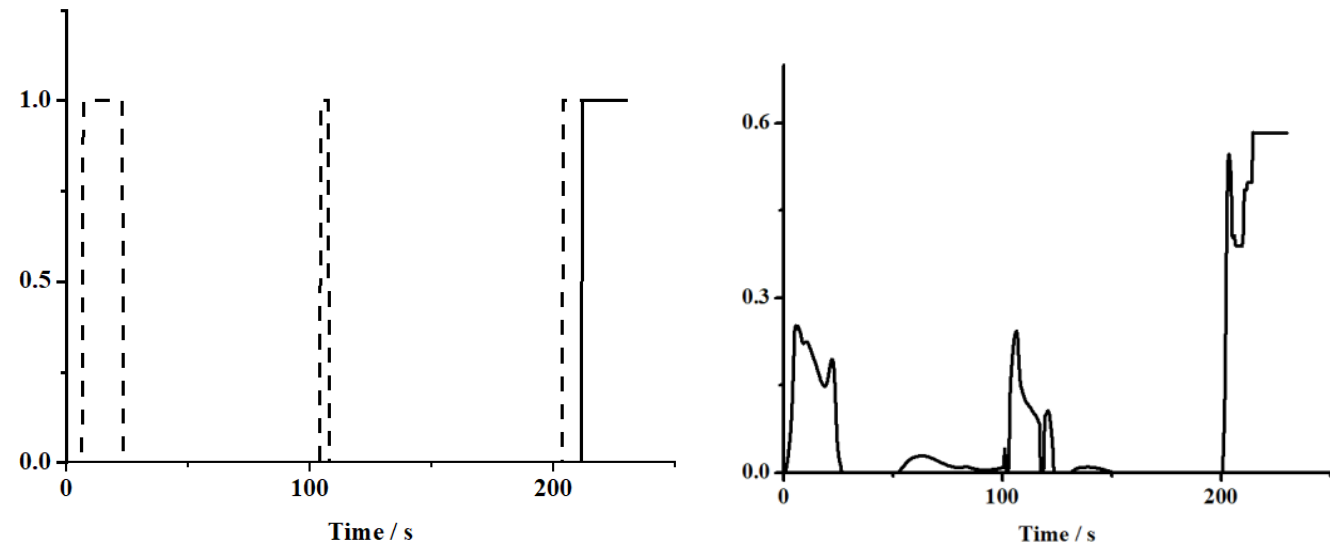

Fig. 18 Diagnosis results with and without predictionFig. 19 Diagnosis result with BP neural network

We considered that the AUV was primarily controlled by only one propeller in the process of turning the bow; for instance, the AUV's right propeller provided the primary part of the thrust, while the left propeller was less involved in the turning left process. Therefore, another simulation test was configured: from $0 \mathrm{~s}$ to $50 \mathrm{~s}$, the AUV was accelerating to $1.0 \mathrm{~m} / \mathrm{s}$, and no fault occurred in the propeller during this process; from $50 \mathrm{~s}$ to $100 \mathrm{~s}$, the AUV was turning $45^{\circ}$ to the left; and the AUV started turning to the right from $100 \mathrm{~s}$. In the simulation, the AUV's left propeller was fault-free at first, but it became faulty starting from $50 \mathrm{~s}$ : the maximum output thrust was $60 \mathrm{~N}$.

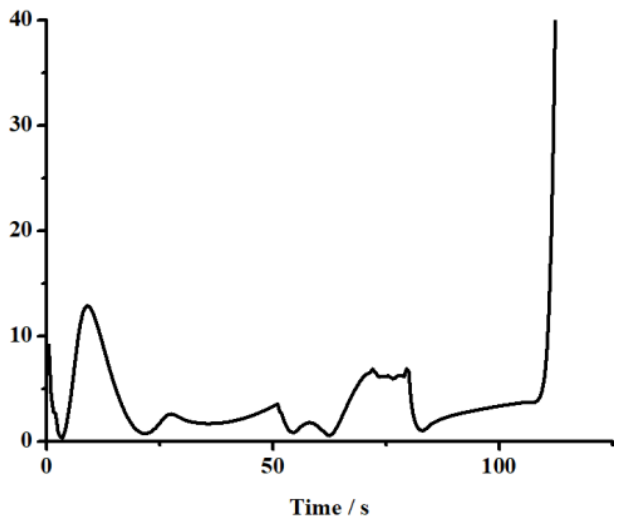

Fig. $20 \mathrm{X}$-axis force loss with $\mathrm{MB}$ algorithm

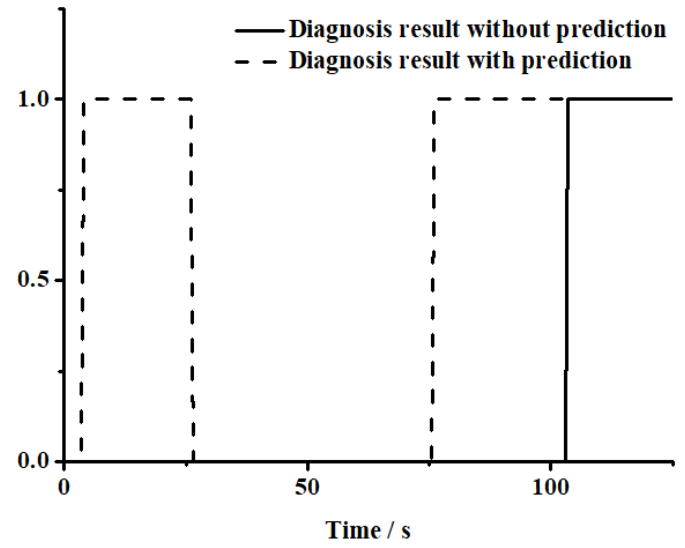

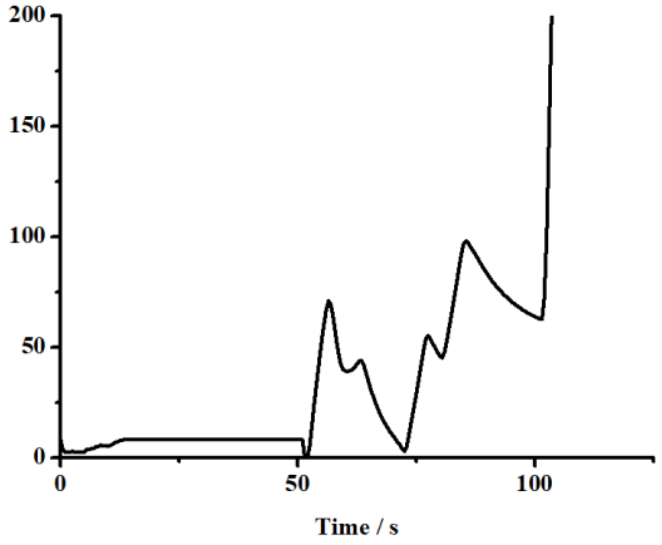

Fig. 21 Yaw moment loss with MB algorithm

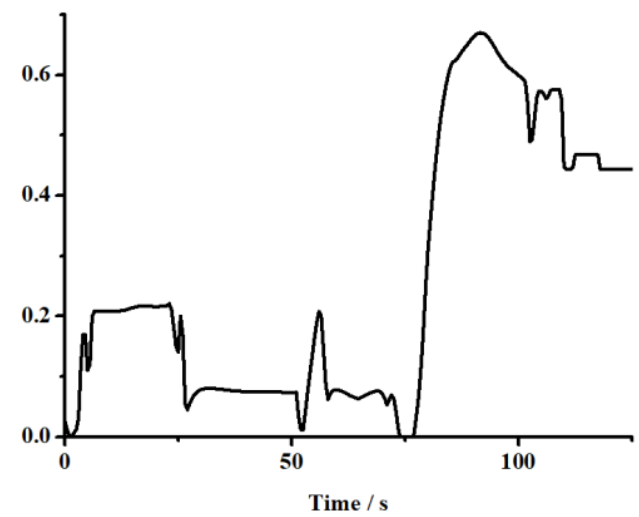

Fig. 22 Diagnosis results with and without predictionFig. 23 Diagnosis result with BP neural network

Fig. 20 to Fig. 23 show that the fault diagnosis algorithm without prediction diagnosed the existence of a fault at $103.5 \mathrm{~s}$, from the MB value in the Z-axis exceeding the threshold, 
whereas the algorithm with prediction diagnosed a fault at $75 \mathrm{~s}$. However, owing to the prediction, from $4 \mathrm{~s}$ to $26 \mathrm{~s}$, a misdiagnosis occurred. The BP neural network diagnosed the existence of a fault at $78.5 \mathrm{~s}$ instead, and a misdiagnosis did not occur.

When the AUV was turning to the left, the yaw moment was primarily provided by the right propeller, and the left one was less involved at the beginning of the process. If the left propeller failed, it was very difficult to accurately judge the problem during this process. The fault diagnosis algorithm did not have the correct estimation of the propeller thrust loss; on the contrary, when the AUV was close to the desired yaw angle, the required thrust of the left propeller increased, the impact of the fault on the AUV's motion state became more obvious, and the presence of the fault was quickly detected.

Finally, we tried to use the real sea trail data to test the performance of the fault diagnosis algorithms. Figures 24 and 25 display a part of the sea trail process with a fault situation. The AUV was configured to sail straight at the velocity of $1.0 \mathrm{~m} / \mathrm{s}$, and then turn the bow to $30^{\circ}$ to continue sailing. From the velocity curve and the yaw angle curve, we observed that the AUV experienced a velocity loss and an unstable change in the yaw angle during the last section of the trail, which corresponded to the thrust loss in the relevant degree of freedom.

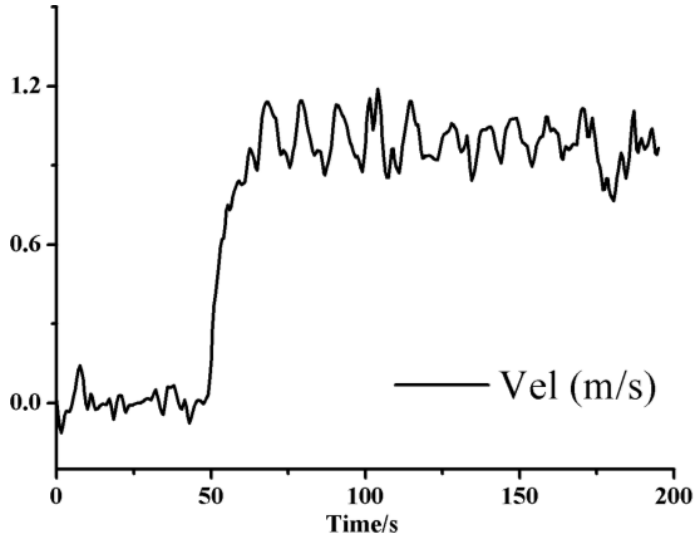

Fig. 24 AUV velocity data in sea trail

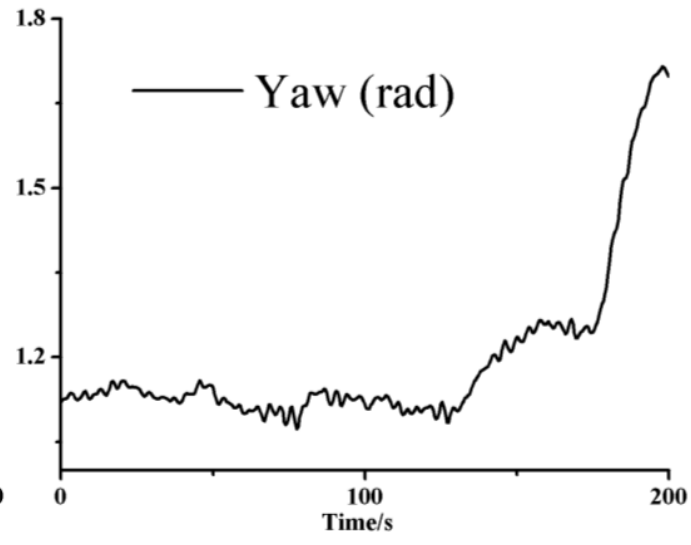

Fig. 25 AUV yaw angle data in sea trail

Figures 26 and 27 compare the results analysed from the sea trail data, using the Gaussian particle filter method [11] and the rank particle filter method. Sea trail data was used by these two kinds of filter methods to estimate the control force and yaw moment losses. Subsequently, the MB algorithm was used to analyse the estimated values during a certain time period. The dash-dot line is the MB value of the Gaussian particle filter and the solid line is the result of the rank particle filter.

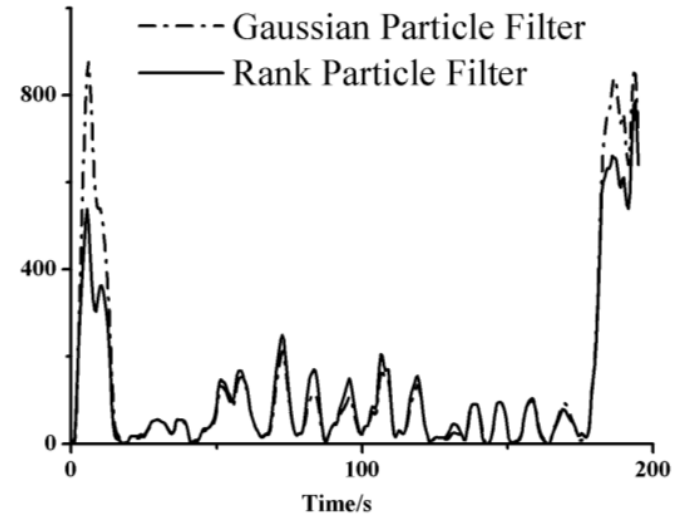

Fig. $26 \mathrm{X}$-axis force loss with $\mathrm{MB}$ algorithm

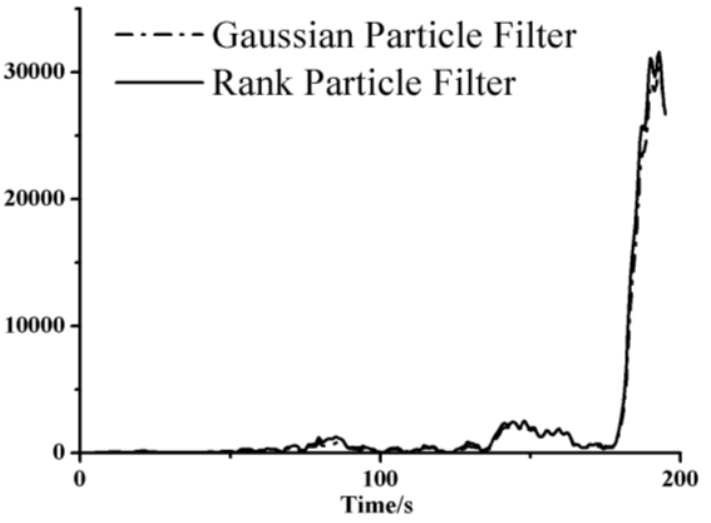

Fig. 27 Yaw moment loss with MB algorithm 
Here, we observed that in the initial stage of releasing the AUV, the Gaussian particle filter method along the $\mathrm{X}$-axis direction has a larger estimation of the control force loss than the rank particle filter method, and the MB value estimated during this stage is close to that at the end of the trail, which indicates that the AUV may be faulty. In contrast, the rank particle filter method estimated the current state of the AUV sooner and more accurately in the initial stage, and also estimated the control force loss quickly at the end of the trail, reducing the possibility of a misdiagnosis.

Environmental interference in the real sea trail and a delayed response in the mechanical devices have also occurred. To determine the threshold value of a fault, several groups of the normal-state data with desired velocities from $0 \mathrm{~m} / \mathrm{s}$ to $1.0 \mathrm{~m} / \mathrm{s}$ during the sea trial process were selected. We obtained the MB values in these various states, and the maximum values were selected to determine the threshold. The AUV had a maximum value of 539 in the Xaxis, 356 in the $\mathrm{Y}$-axis, and 2518 in the $\mathrm{Z}$-axis. At the same time, in the training process of the BP neural network, the expected diagnostic value of the turning process with acceleration was set as 0.3 and the expected diagnostic value of the accelerating process was set as 0.2 . Using this method, the trained BP neural network could be used to detect the existence of a fault; the fault diagnosis process is shown in Figure 28.

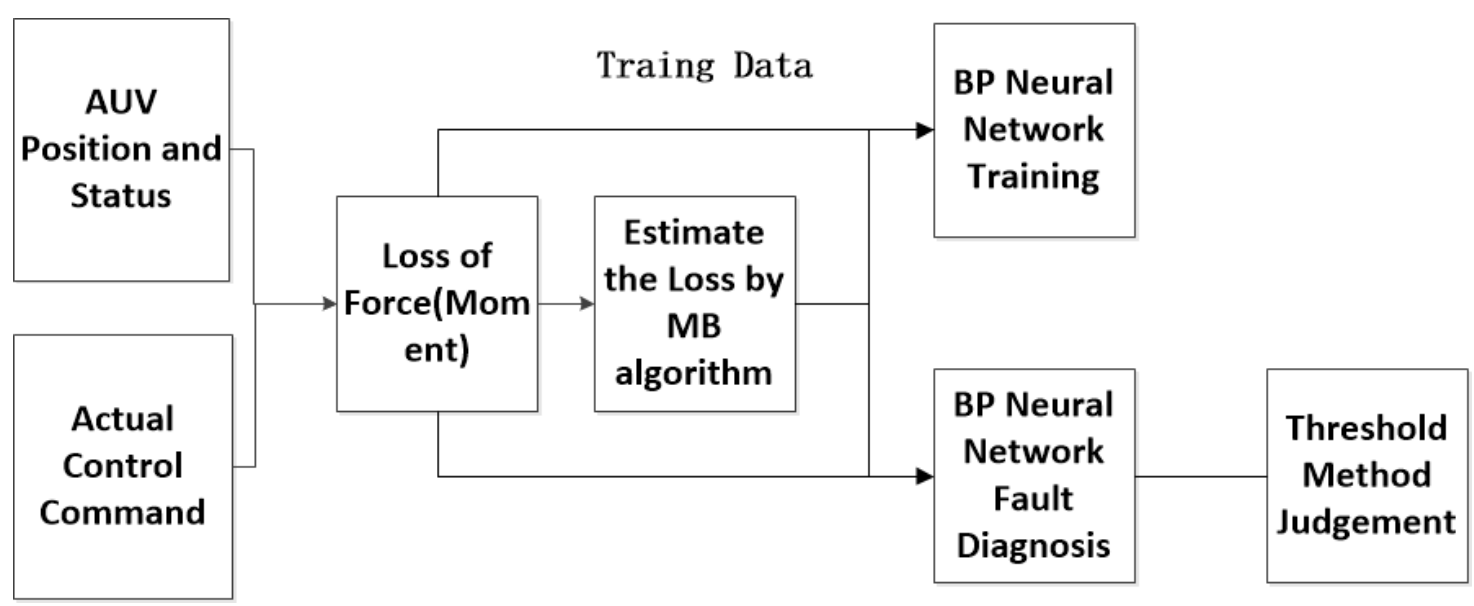

Fig. 28 Fault diagnosis simulation using the actual sea trail data

Figure 29 shows the comparison between the fault diagnosis results with and without prediction. It is known that a certain deviation exists between the actual model of an AUV in the sea trail and the hydrodynamic model in the simulation. Over the course of the experiment, the fault diagnosis algorithm without prediction diagnosed a fault at $180.5 \mathrm{~s}$. Further, the algorithm with prediction detected a fault at $178.5 \mathrm{~s}$, but misdiagnoses still occurred. This was also due to the amplified loss that caused the MB value to be greater than the threshold, and the fault threshold was kept as before. In Figure 30, from the diagnostic result by the BP neural network, we observed some delays between the control command and the response of the actuator when the AUV moves from the initial position at around $50 \mathrm{~s}$. During this stage, a relatively greater diagnostic value compared with the rest of the normal conditions existed. From $125 \mathrm{~s}$ to $178 \mathrm{~s}$, the average absolute value of the diagnostic results is greater than the average absolute value of the previous $50 \mathrm{~s}$, because the AUV was turning its bow, and there was a larger change in the $\mathrm{Y}$-axis direction. A certain degree of increase in the force loss would occur in the Y-axis, causing the diagnostic value of the BP neural network to vibrate. Finally, the diagnostic value at $179 \mathrm{~s}$ exceeds the threshold of 0.3 , implying that the AUV's propeller was faulty. 


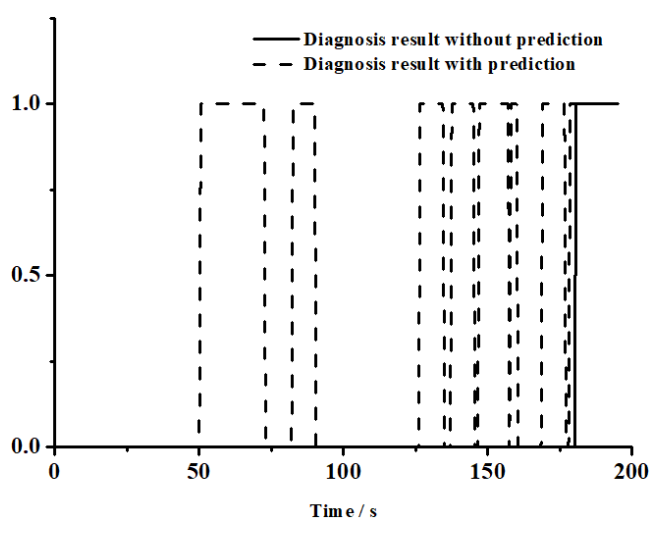

Fig. 29 Fault diagnosis results comparison

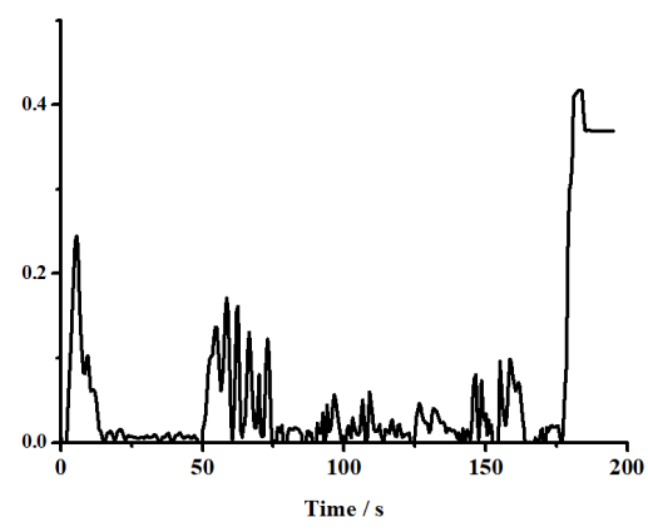

Fig. 30 BP neural network diagnosis result

\section{Conclusions and Future Work}

1. The simulation results show that the rank particle filtering method can be applied to fault diagnosis, and the control force (moment) loss can be analysed through the MB algorithm with the sliding time window.

2. The proposed algorithm can detect the fault in advance through the multi-step-ahead prediction process, and the misdiagnosis caused by using only the loss in a single degree of freedom can be reduced using the BP neural network.

3. The noise that exists in the sea trail environment creates a mostly non-Gaussian case. The rank particle filter method is suitable for the actual state estimation of an AUV and helps to improve the accuracy of the diagnosis.

4. The next step is to increase the real-time ability of the fault diagnosis algorithm, and complete the real-time diagnosis of failure of an AUV in open water, or under conditions where currents exist.

\section{Acknowledgement}

This work was supported by the National Key R\&D Program of China (No. 2017YFC0305700), National Natural Science Foundation of China (No. 51609047, 51509057), China Postdoctoral Science Foundation (No. 2017M621250).

\section{REFERENCES}

[1] Stilinović N, Marković M, Mišković N, et al. MECHANICAL DESIGN OF AN AUTONOMOUS MARINE ROBOTIC SYSTEM FOR INTERACTION WITH DIVERS[J]. Brodogradnja Časopis Brodogradnje I Brodograđevne Industrije, 2016, 67(3).

[2] Xiang X, Yu C, Lapierre L, et al. Survey on Fuzzy-Logic-Based Guidance and Control of Marine Surface Vehicles and Underwater Vehicles[J]. International Journal of Fuzzy Systems, 2017(1):1-15. Published online-first. https://doi.org/10.1007/s40815-017-0401-3.

[3] Xiang X, Liu C, Su H, et al. On decentralized adaptive full-order sliding mode control of multiple UAVs[J]. Isa Transactions, 2017,71:196-205. https://doi.org/10.1016/j.isatra.2017.09.008

[4] Martinović D, Tudor M, Bernečić D. An Analysis of Starting Points for Setting Up a Model of a More Reliable Ship Propulsion[J]. Brodogradnja, 2011, 62(3):249-258.

[5] Momma H, Watanabe M, Hashimoto K, et al. Loss of the full ocean depth ROV Kaiko - Part 1: ROV Kaiko - A review. In: Proceedings of 14th International Offshore and Polar Engineering Conference[C]// 2004.pp. 23-28

[6] Showstack R. Unmanned Research Vessel Lost on Deep Sea Dive[J]. Eos Transactions American Geophysical Union, 2014, 95(20):168-168. https://doi.org/10.1002/2014EO200004. 
[7] Gafurov S A, Klochkov E V. Autonomous Unmanned Underwater Vehicles Development Tendencies [J]. Procedia Engineering, 2015, 106:141-148. https://doi.org/10.1016/j.proeng.2015.06.017.

[8] Mai B L, Choi H S, Seo J M, et al. Development and control of a new AUV platform[J]. International Journal of Control Automation \& Systems, 2014, 12(4):886-894. https://doi.org/10.1007/s12555-0120385-6.

[9] Xiang X, Yu C, Zhang Q. On intelligent risk analysis and critical decision of underwater robotic vehicle[J]. Ocean Engineering, 2017, 140:453-465. https://doi.org/10.1016/j.oceaneng.2017.06.020

[10] Liu Q, Zhu D. Fault-tolerant Control of Unmanned Underwater Vehicles with Continuous Faults: Simulations and Experiments[J]. International Journal of Advanced Robotic Systems, 2010, 6(4):301-308.

[11] Sonoda T, Fujii N, Nishida Y, et al. Development of Self-Diagnosis System of an Autonomous Underwater Vehicle Tuna-Sand 2[J]. Self, 2017, 4(3): 8.

[12] Barisic M, Vasilijevic A, Nad D. Sigma-point Unscented Kalman Filter used for AUV navigation[C]// Control \& Automation. IEEE, 2012:1365-1372.

[13] Filaretov V, Zhirabok A, Zuev A, et al. Development of subsystem of accommodation to faults for dead reckoning system of autonomous underwater vehicles[J]. ARPN Journal of Engineering and Applied Sciences, 2017, 12(4): 1309-1314.

[14] Chu Z Z, Zhang M J. Fault reconstruction of thruster for autonomous underwater vehicle based on terminal sliding mode observer[J]. Ocean Engineering, 2014, 88(5):426-434. https://doi.org/10.1016/j.oceaneng.2014.06.015.

[15] Sun Y, Ran X, Li Y, et al. Thruster fault diagnosis method based on Gaussian particle filter for autonomous underwater vehicles[J]. International Journal of Naval Architecture and Ocean Engineering, 2016, 8(3): 243-251. https://doi.org/10.1016/j.ijnaoe.2016.03.003

[16] David F. Bauer. Constructing Confidence Sets Using Rank Statistics[J]. Journal of the American Statistical Association, 1972, 67(339):687-690. https://doi.org/10.1080/01621459.1972.10481279

[17] Huimin F U, Qiang X, Lou T S, et al. Rank Particle Fitler[J]. Journal of Mechanical Strength, 2014(6):894-898.

Submitted: $\quad$ 13.10.2017. Jiayu He

$\begin{array}{ll} & \text { Ye Li, liyeheu103@163.com } \\ \text { Accepted: } & \text { 26.01.2018. } \\ & \text { Yanqing Jiang } \\ & \text { Li An } \\ & \text { Science and Technology on Underwater Vehicle Laboratory } \\ & \text { Harbin Engineering University, Harbin 150001, China }\end{array}$

